\title{
Microprocessor-Based Precision Speed Measurement
}

\author{
Ratil Hasnat Ashique ${ }^{1}$ \\ ${ }^{I}$ Primeasia University, Department of EEE ,12,Kamal Atartuk Avenue,Banani,Dhaka.
}

\begin{abstract}
An optical disc and microprocessor (i-8086 $\varepsilon$ i-8087)- based speed measurement scheme is presented in this paper. Improvement of accuracy and minimization of phase angle error are achieved by varying sampling time in the speed detection algorithm. The scheme was implemented as an integral component of a vector control scheme for induction motors and was found to work effectively.
\end{abstract}

Keywords: Instrumentation, Microcontroller, Speed detection, Phase angle error, Vector Control, Automation, Optical Encoder, Timer.

\section{Introduction}

Most of the industrial drives in application, now-a-bays, use closed loop speed control scheme. For this, the instantaneous speed of the drive need be accurately sensed of detected. Specially, if the drive is a high performance servo type, the efficacy of the control scheme becomes highly dependent on the accuracy of the detected speed with respect to its actual instantaneous speed which is normally subjected to variations. An ordinary (dc) based detection scheme fails to meet this requirement due to its inherent nonlinearity [1]. Alternative to this are: (i) multi pole ac tacho generator, (ii) optical encoder microprocessor syscem. The first and second devices are rarely used because of their costs. Since the major control schemes at present use microprocessor as the key device of the digital control scheme, major researchers [24] show interest in the optical disc/ encoder. microprocessor-based speed detection scheme. In the optical encoder microprocessorbased system, the average speed of the drive over an interval is detected. The variables, in this method, are: the number of encoder pluses (m) and the duration of these pluses $(\mathrm{T})$. There are distinctly three methodologies found in [2]. These are (i) the m-method, (ii) the T-method and (iii) the $\frac{m}{T}$ method. For variable speed drives, the $\frac{m}{T}$ method is the best choice as its accuracy is marginally affected by the range of measured speed. To improve the accuracy of this method, an adaptive optimization technique is suggested in [3] which automatically adjusts the sampling time according to the width of the encoder pulse. For highly dynamic or vibratory systems, accuracy at constant speed as well as phase angle error between instantaneous speed and the detected moving average speed are to be considered with equal importance for improving the stability in performance [4-5].

This paper presents a technique in which both the above mentioned criteria are dealt with equal importance. For accuracy of the measured speed, the microprocessor has to wait in a loop so that integral number of encoder pulse is counted in detecting the time. The pre-detection interval is proposed to keep at a suitable value and is estimated by the reference and actual speed of the drive system for realization of the nature of dynamic condition.

\section{Principle of Operation}

The optical encoder-microprocessor-based scheme stands on counting the integral number of optical pulses and time required for these pulses. For measuring time, fixed frequency on board programmable counters are used. If $\mathrm{n}$ is the difference in counter reading and $\mathrm{f}_{\mathrm{c}}$ is the frequency of the programmable counter, then the detected time is computed as follows:

$\mathrm{T}=\frac{\mathrm{n}}{\mathrm{f}_{\mathrm{c}}}$

Normally, the frequency of the counter is very high in comparison with the optical encoder. The accuracy in the detected time is thus ascertained by creating delay when the microprocessor goes to read the counter register so that the reading corresponds to exactly integral number of encoder pulses. The preset detection interval $(\mathrm{Tp})$ and exact detection interval $(\mathrm{T})$ along with encoder pulse and counter clock are shown in

Fig. 1. The correction in time is $\Delta \mathrm{T}$ as indicated in this figure. 


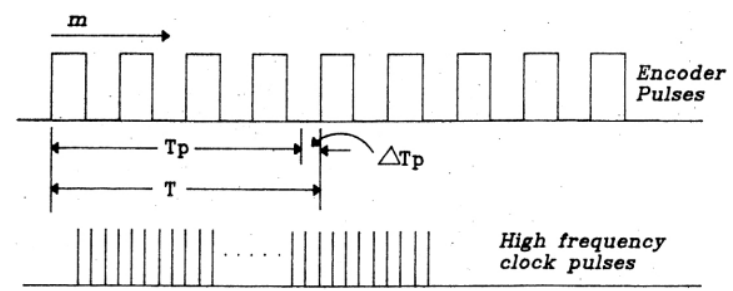

Fig. 1: Adjustment of Preset Detection Time for Intregral Number of Encoder Pulses

Let the optical disc generates $M$ pulses per rotation of the shaft, the average speed in rpm over the interval, if $\mathrm{m}$ encoder pulses are detected for the interval $\mathrm{T}$, is given as

$$
\mathrm{N}_{\mathrm{r}}=\frac{60}{\mathrm{M}} \cdot \frac{\mathrm{m}}{\mathrm{T}}
$$

i.e.

$$
\mathrm{N}_{\mathrm{r}}=\frac{60 \mathrm{mf}_{\mathrm{c}}}{\mathrm{nM}} \cdot \frac{\mathrm{m}}{\mathrm{T}}
$$

In Eqn. 3, $\mathrm{M}$ and $\mathrm{f}_{\mathrm{C}}$ are constants and for usual range of operation hardly an error is introduced in determining $\mathrm{m}$, so that the resolution [2] is expressed as

$\mathrm{Q}_{\mathrm{n}}=\frac{60 \mathrm{mf}_{\mathrm{c}}}{\mathrm{M}} \cdot \frac{1}{\mathrm{n}(\mathrm{n}-1)} \approx \frac{60 \mathrm{mf}_{\mathrm{c}}}{\mathrm{M} \cdot \mathrm{n}^{2}}$

Eqn. 4 indicates that if $\mathrm{n}$ is high, then the resolution $\left(\mathrm{Q}_{\mathrm{n}}\right)$ is better but if $\mathrm{n}$ becomes low, then the resolution $\left(\mathrm{Q}_{\mathrm{n}}\right)$ becomes poor.

\section{Implementation}

The pulse generation and shaping circuits of the encoder consists of infra-red LEDs as emitter and receiver of opto-electrical signals and are placed in head to head fashion to the opposite sides of the encoding disc as show in Fig 2.

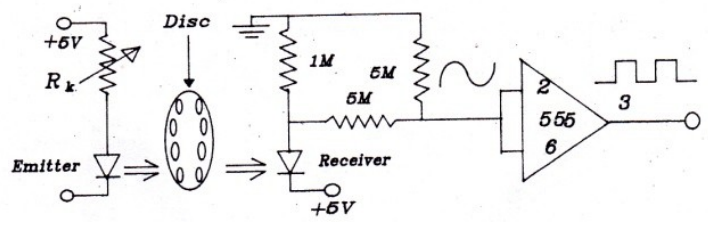

Fig. 2: Pulse Generation Circuti for the Optical Encoder

The aluminum encoder disc in fig. 2. contains 128 slots. As the disc rotates, a voltage swing appears at the output circuit of the receiver. The level of the swing is adjusted by varying $\mathrm{Rk}$ at the full speed of the drive so that the levels are well below $1 / 3 \mathrm{~V}_{\mathrm{cc}}$ and well above $2 / 3 \mathrm{~V}_{\mathrm{cc}}$. The signal is then applied to the level comparator that generates sharp square wave pulses. The optical encoder pulse is interfaced with a particular data bit of the programmable digital (ppi-8255A) port and also applied as clock of counter \#1 of the programmable interval timer (8253). Another counter of i-8253 (counter \#2) is clocked by the system peripheral clock of $2.386 \mathrm{MHz}$. The Microfriend(MF8679) micro computer system consists of intel-8086 up as CPU and i-8087 as mathcoprocessor (NDP). On board are interfaced with all the above mentioned programmable peripherals. All necessary computations are carried out in teal format using the NDP. Both the counters of i-8253 are programmed in mode 2 and loaded with FFFFH initially.

\section{Accuracy, Constraints and Optimization}

Optimization of the preset sampling interval is required for minimizing error in detecting speed by an optical encoder for the over all range of operation of the drive. It is worth mentioning that in most practical control system, the microprocessor computes average speed as an integral part of the control law implementation. In this, it counts the number of optical pulses (m) from an optical disc and computes time using a fixed high frequency peripheral timer (counter). In counting time tor an integral number of optical disc pulses, the microprocessor has to wait in a loop and the maximum possible error in time introduced is about 10.04 u.sec. If the preset detection interval is large this error has negligible effect on steady state result. It is shown in [5] that an 8-bit optical encoder produces a speed error of $0.6 \mathrm{rps}$ (36rpm) to measure $1500 \mathrm{rpm}$. The proposed method implemented with a 16-bit counter may introduce a speed error of $0.0997 \mathrm{rps}$ (5.98rpm) to $0.0133 \mathrm{rps}$ $(0.8 \mathrm{rpm})$ at the sampling times of $2.5 \mathrm{msec}$ and $30 \mathrm{msec}$ respectively. The corresponding acceleration errors are 
$39.87 \mathrm{rps}^{2}$ and $0.444 \mathrm{rps}^{2}$ respectively, which are very small in comparison with the results $\left(120 \mathrm{rps}^{2}\right)$ in [5]. The scheme when used for sensing speed of a Field Oriented drive system is thus free from generating speed oscillations under steady state conditions [5]. On the other hand, the phase shift between the instantaneous speed and the average speed increases with the increase in detection interval. This aspect is demonstrated with the help of the following sinusoidal speed profile consistent with the speed response of an induction motor an:

$$
\mathrm{Ni}=900+600 \sin (\pi t)
$$

For a constant detection interval of $20 \mathrm{msec}$, the instantaneous speed, the detected speed and speed error profiles are shown in Fig. 3. It is observed from these curves that the phase shift between the instantaneous speed the detected speed produces error which has a maximum value when fast changes occur in the instantaneous speed. The phase angle error increases with the detection interval (sampling time) and it is represented in Fig. 4. The maximum possible error introduced by the proposed method for the bit test loop introduces error under steady state operation also. This error decreases with increase of sampling time and it is demonstrated in Fig. 4. Considering these aspects the sampling time in kept at a low value while sharp change in speed occurs.

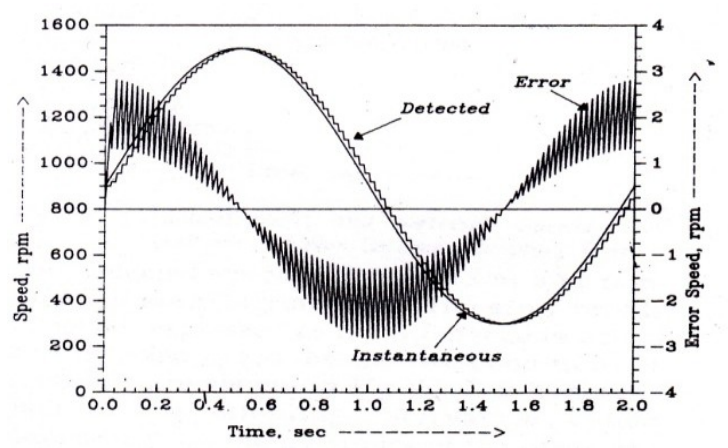

The Instantaneous all Detected Speed.Speed Error profiles for a Constant Sampling Time.

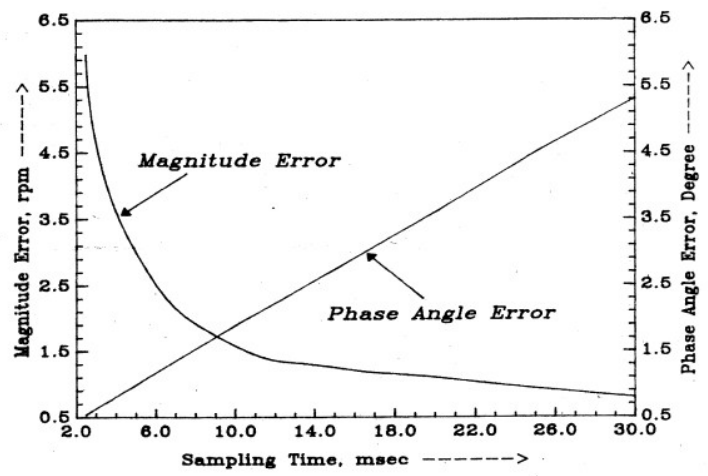

Fig. 4: Magnitude and Phase Angle Error Curves for the Proposed Method

The proposed method uses the speed measurement scheme for implementing the vector control of a CSI-fed induction motor drive. In this, the microprocessor controls the firing of converter and inverter thyristors. Time required for doing these controls is about $2 \mathrm{msec}$. In an Auto-Sequentially Commutated Inverter (ASCI), six pulses per cycle are generated for inverter firing. The minimum preset detection interval is selected as the interval between the firing instances of two consecutive interval thyristors and for $50 \mathrm{~Hz}$ operation, it is 3.33 msec. For a 16 bit counter of the programmable interval timer (8253), the minimum speed that could be detected by the counter with $2.386 \mathrm{MHz}$ clock is about $150 \mathrm{rpm}$. However, if tow counters used for having 32 bit counting, then very low speeds could be measured. If the speed of the drive changes, the detection interval also changes and for higher speed range, the detection interval is smaller that may increases steady state error. The accuracy can be improved by dividing the useful speed range of the drive into six segments and a coefficient $\mathrm{k}$ (for $200-400 \mathrm{rpm}$ : $\mathrm{k}=1$ for $401-600 \mathrm{rpm}$ : $\mathrm{k}=2$.............., for $1201-1400 \mathrm{rpm}$ : $\mathrm{k}=6$ ) corresponding to the number of firing intervals of the consecutive inverter thyristors is proposed for operation under steady state. However, if the drive is changing speed at a fast rate then the value of $\mathrm{k}$ is constrained to 1 . This is done by comparing the difference in reference and actual speed. To visualize this aspect, an exponential speed rise profile similar to the starting speed response of an induction motor drive was considered. The speed was detected with uniform sampling interval $(20 \mathrm{msec}$ ) and variable sampling interval (varying from $4 \mathrm{msec}$ to 24 $\mathrm{msec}$ ). The coefficient ( $\mathrm{k}$ ) for changing the sampling interval was incorporated by comparing the actual speed with the reference (final) speed. The curves in Fig. 5(a) indicate the variation of the actual 


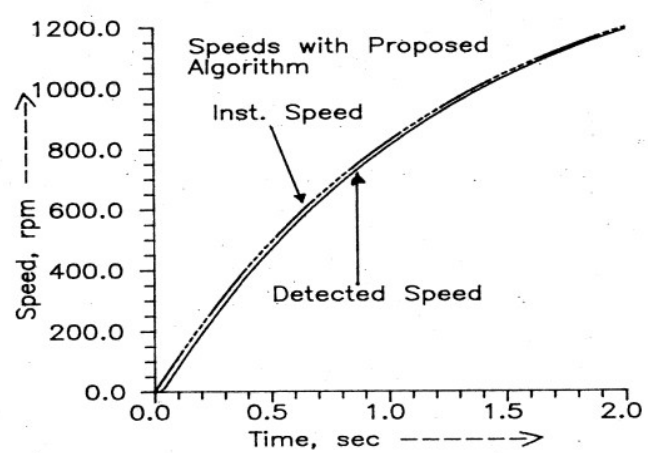

Fig. 5 (a) Instantaneous and Detected Speeds with Uniform and Non-Uniform Sampling Time

and detected speeds with uniform and nonuniform sampling intervals. The error profile in speed, expressed as the difference in actual and detected speed, by the two conditions of sampling are shown in Fig (5). It is observed that if the sampling interval is varied depending on the dynamics of the drive, the error can be limited to narrow range with optimized time saving operation of the microprocessor.

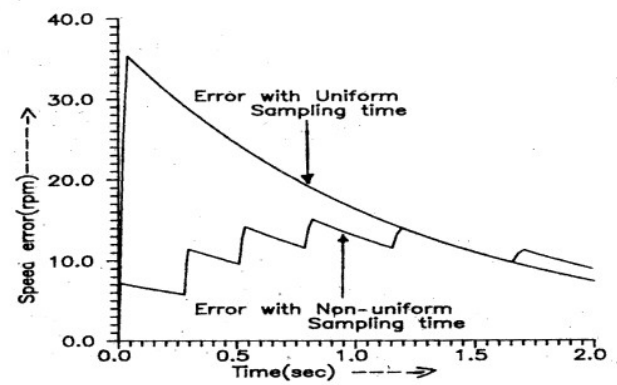

Fig. 5 (b): Comparison of Speed Error for Uniform and NonOUniform Sampling Time

\section{Experimental Verification}

The proposed speed measurement scheme was tested for determining its accuracy at different speeds of a separately excited dc motor and the detected speed was compared with that of a digital tachometer under steady-state conditions. A close agreement between the two sets of measured values in the useful range was observed. A comparison of the steady state values ot the precision tachometer reading those of the detected speed by the proposed method and the corresponding one with tacho generator readings are shown in Fig. 6.. It is observed that at higher values of the speed the tacho generator shows error in its generated voltage (converted to speed).

To test the efficacy of the proposed method in detecting instantaneous speed, the speed rise profile of a $\mathrm{dc}$ motor starting from standstill was recorded using a de tacho generator and the proposed system.

These characteristics are shown in Fig. 7. A very close speed rise profile observed from both the systems in detecting speed during acceleration. A slight deviation at the final speed (1000) rpm) is which indicate the

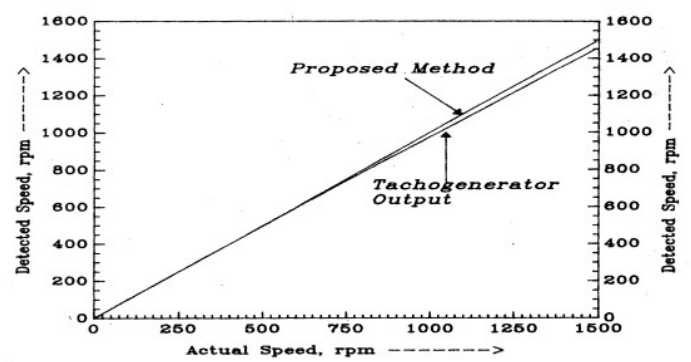

Fig. 6: Comparision of Speed Detection by Proposed Method and Tachogenerator Method

effectiveness of the scheme noted in Fig. 7 which is due to nonlinearity of the dc tachogenerator. Finally, as an integral part of the vector control scheme, the speed rise from standstill condition to the steady state of the drive is shown in Fig. 8. 


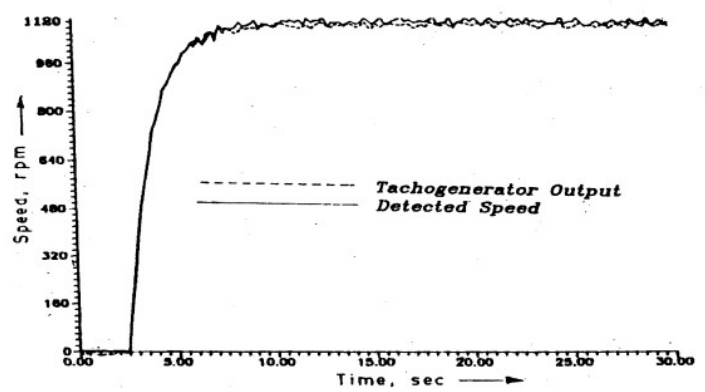

Fig. 7: Detection of Instantaneous Speed by the Imposed Method and Comparison with the Tacho generator Output (Up to Steady State)

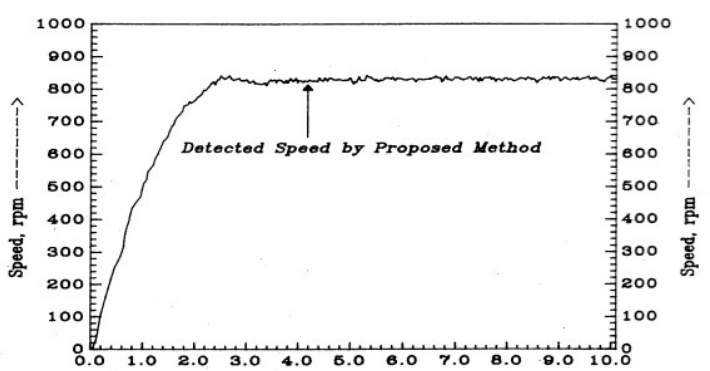

Fig. 8: the Speed rise Curve of an Inductive Motor under Vector Control Scheme using the Proposed Speed Detection Method.

\section{Conclusion}

The speed measurement scheme, presented in this paper, is a simple and a versatile one. The method requires minimum hardware components for its implementation. For medium range of speed, the scheme is found to work satisfactorily. It is observed that accuracy in detected speed is improved by varying sampling interval However, time lag exists between the detected and actual speed which requires instantaneous speed estimation algorithm. For very high speed, the time constant of the infra-red LED receiver constraints the pulse generation and limits its use.

\section{References}

[1] T. Kume And T. Iwakane, "High Performance Vector Controlled AC Motor Drives: Applications and New Technologies", IEEE Transactions on Industry Applications., Vol. IA-23,PP 172- 180, (1987)

[2] T. Ohmae, T. Matsuda, K. Kamiyama and M. Tachikawa, "A Microprocessor Controlled High Accuracy Wide-Range Speed Regulator for Motor Drives”, IEEE Transactions on Industrial Electronics, Vol. IE-29, PP 203-211, (1982).

[3] N. Chauduri, S. Ghosh, and A.M. Ghosh "Wide-Range Precision Speed Measurement with Adaptive Optimization Using Microcomputer", IEEE Transaction on Industrial Electronics, Vol. IE-30, pp 369-373, (1983).

[4] K. Saito, K. Kamiyama, T. Ohmae and Matsuda, "A Microprocessor-Controlled speed Regulator with Instantaneous Speed Estimation for Motor Drives" IEEE Transactions on Industrial Electronics, Vol IE-35, PP95-99, (1988).

[5] K. Jelassi, B. de Fornell and M.P. David, Quantization and Discretization on the Field Oriented Asynchronous Drive", ICEM '90, MIT USA, PP 550-555, (1990).

\section{Authors profile}

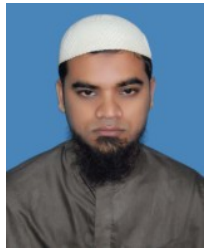

Ratil Hasnat Ashique received the B.Sc degree in Electrical Engineering from Bangladesh University of Engineering and Technology in 2011 and a MSc Student in the same university. $\mathrm{He}$ is now working as a lecturer in Primeasia University, Dhaka at the department of EEE. He also worked as a Lecturer at Presidency university in the department of ECE. 